\title{
DEIMOS: A beamline dedicated to dichroism measurements in the 350-2500 eV energy range
}

P. Ohresser, E. Otero, F. Choueikani, K. Chen, S. Stanescu, F. Deschamps, T. Moreno, F. Polack, B. Lagarde, J.-

P. Daguerre, F. Marteau, F. Scheurer, L. Joly, J.-P. Kappler, B. Muller, O. Bunau, and Ph. Sainctavit

Citation: Review of Scientific Instruments 85, 013106 (2014); doi: 10.1063/1.4861191

View online: https://doi.org/10.1063/1.4861191

View Table of Contents: http://aip.scitation.org/toc/rsi/85/1

Published by the American Institute of Physics

\section{Articles you may be interested in}

A combined DFT and restricted open-shell configuration interaction method including spin-orbit coupling:

Application to transition metal L-edge X-ray absorption spectroscopy

The Journal of Chemical Physics 138, 204101 (2013); 10.1063/1.4804607

Requirements for $\mathrm{x}$-ray magnetic circular dichroism on paramagnetic biological systems and model compounds Review of Scientific Instruments 73, 1649 (2002); 10.1063/1.1445829

Spin crossover in $\mathrm{Fe}$ (phen) 2 (NCS) 2 complexes on metallic surfaces

The Journal of Chemical Physics 146, 092312 (2017); 10.1063/1.4973511

High-efficiency in situ resonant inelastic x-ray scattering (iRIXS) endstation at the Advanced Light Source Review of Scientific Instruments 88, 033106 (2017); 10.1063/1.4977592

Natural widths of atomic $\mathrm{K}$ and $\mathrm{L}$ levels, Ka X-ray lines and several KLL Auger lines Journal of Physical and Chemical Reference Data 8, 329 (1979); 10.1063/1.555595

Material/element-dependent fluorescence-yield modes on soft X-ray absorption spectroscopy of cathode materials for Li-ion batteries

AIP Advances 6, 035105 (2016); 10.1063/1.4943673

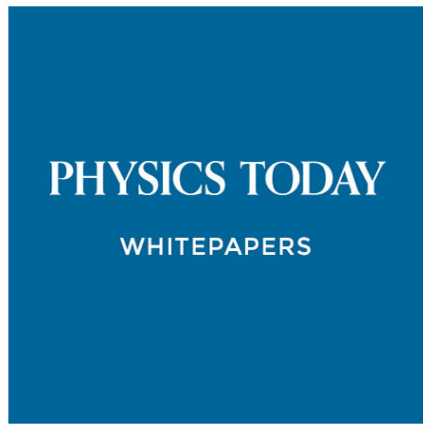

MANAGER'S GUIDE

Accelerate R\&D with

Multiphysics Simulation
READ NOW

PRESENTED BY

○OMSOL 


\title{
DEIMOS: A beamline dedicated to dichroism measurements in the 350-2500 eV energy range
}

\author{
P. Ohresser, ${ }^{1, \text { a) }}$ E. Otero, ${ }^{1}$ F. Choueikani, ${ }^{1}$ K. Chen,${ }^{1}$ S. Stanescu, ${ }^{1}$ F. Deschamps, ${ }^{1}$ \\ T. Moreno, ${ }^{1}$ F. Polack, ${ }^{1}$ B. Lagarde, ${ }^{1, b)}$ J.-P. Daguerre, ${ }^{1, c)}$ F. Marteau, ${ }^{1}$ F. Scheurer, ${ }^{2}$ \\ L. Joly, ${ }^{2}$ J.-P. Kappler, ${ }^{1,2}$ B. Muller, ${ }^{2}$ O. Bunau, ${ }^{1,3}$ and Ph. Sainctavit ${ }^{1,3}$ \\ ${ }^{1}$ Synchrotron SOLEIL, L'Orme des Merisiers, Saint-Aubin - BP 48, 91192 Gif-sur-Yvette, France \\ ${ }^{2}$ Institut de Physique et Chimie des Matériaux de Strasbourg, UMR 7504 UdS-CNRS, \\ 67034 Strasbourg Cedex 2, France \\ ${ }^{3}$ Institut de Minéralogie et de Physique des Milieux Condensés, CNRS UMR 7590, \\ Université Pierre et Marie Curie, 75252 Paris Cedex 5, France
}

(Received 1 October 2013; accepted 18 December 2013; published online 21 January 2014)

The DEIMOS (Dichroism Experimental Installation for Magneto-Optical Spectroscopy) beamline was part of the second phase of the beamline development at French Synchrotron SOLEIL (Source Optimisée de Lumière à Energie Intermédiaire du LURE) and opened to users in March 2011. It delivers polarized soft $\mathrm{x}$-rays to perform x-ray absorption spectroscopy, x-ray magnetic circular dichroism, and x-ray linear dichroism in the energy range 350-2500 eV. The beamline has been optimized for stability and reproducibility in terms of photon flux and photon energy. The main endstation consists in a cryo-magnet with 2 split coils providing a $7 \mathrm{~T}$ magnetic field along the beam or $2 \mathrm{~T}$ perpendicular to the beam with a controllable temperature on the sample from $370 \mathrm{~K}$ down to 1.5 K. (C) 2014 AIP Publishing LLC. [http://dx.doi.org/10.1063/1.4861191]

\section{INTRODUCTION}

The DEIMOS (Dichroism Experimental Installation for Magneto-Optical Spectroscopy) beamline is the beamline dedicated to soft x-ray dichroic measurements at SOLEIL (Source Optimisée de Lumière à Energie Intermédiaire du LURE). Such measurements are obtained by comparing two x-ray absorption (XA) spectra recorded for different sample states and/or different light polarizations. The photon source is constituted by two undulators installed on the I07-m medium straight section of the SOLEIL storage ring: a 1.65 $\mathrm{m}$ long Apple-II helical undulator ${ }^{1}$ with a period of $52.4 \mathrm{~mm}$, providing circularly polarized light (left and right) over the full energy range 260-2500 eV (with the first harmonic covering the range $260-1300 \mathrm{eV}$ ) and horizontal and vertical linearly polarized light from $350 \mathrm{eV}$ to $2500 \mathrm{eV}$. The second undulator is an hybrid electromagnet/permanent magnet helical undulator (EMPHU) ${ }^{2}$ with a period of $65.0 \mathrm{~mm}$ optimized for a fast switching $(5 \mathrm{~Hz})$ of the circular polarized light, with no access to the pure linear horizontal polarization. The $350-2500 \mathrm{eV}$ energy range was chosen to cover the principal chemical elements linked to materials showing magnetic properties: the first $(3 d)$ and second $(4 d)$ row transition metal $L$-edges, the rare earth element $M$-edges and carbon, nitrogen, oxygen, and sulphur K-edges which are particularly interesting in the field of molecular magnetism. ${ }^{3}$ This beamline is devoted primarily to the studies of magnetic and electronic

a) Author to whom correspondence should be addressed. Electronic mail: philippe.ohresser@synchrotron-soleil.fr

b) Present address: Brain Physiology Lab, CNRS UMR 8118, Université Paris Descartes, 75270 Paris Cedex 6, France.

c) Present address: Plateforme Régionale d'Innovation BOIS HD, Atlanpôle, 44306 Nantes cedex 3, France. states of nanoscale structures. XMCD (X-ray Magnetic Circular Dichroism) and XMLD (X-ray Magnetic Linear Dichroism) as well as XNCD (X-ray Natural Circular Dichroism) and XNLD (X-ray Natural Linear Dichroism) are indeed very sensitive techniques allowing to probe minute amounts of matter ${ }^{4,5}$ with the unique capability to provide information with chemical and orbital selectivity by measuring the appropriate absorption edge. Moreover, for some edges, XMCD can also be used to obtain quantitative information on the spin and orbital magnetic moments using specific sum rules. ${ }^{6}$ As the magnetic properties, particularly in nanoscale objects, are exacerbated by large magnetic field and low temperature, the main end-station is a superconducting cryo-magnet producing $7 \mathrm{~T}$ along the beam or $2 \mathrm{~T}$ perpendicular to the beam; the temperature on the sample can be set from $1.5 \mathrm{~K}$ to $370 \mathrm{~K}^{7}$

In this paper, we will first present the optical layout of the beamline along with some results from the commissioning of the monochromator. Then we will concentrate on describing the technical developments specifically made to address our beamline demanding characteristics. These developments are (i) the control-command with the implantation of a fast XA spectra data acquisition; (ii) the hybrid undulator (EMPHU), and (iii) the use of a multilayer grating designed to reach the upper energy range (up to $2500 \mathrm{eV}$ ).

\section{BEAMLINE IMPLEMENTATION}

The schematic layout of the beamline, from the source to the experimental end-station, is shown in Figure 1; the main parameters are summarized in Table I (sources) and Table II (optics). 


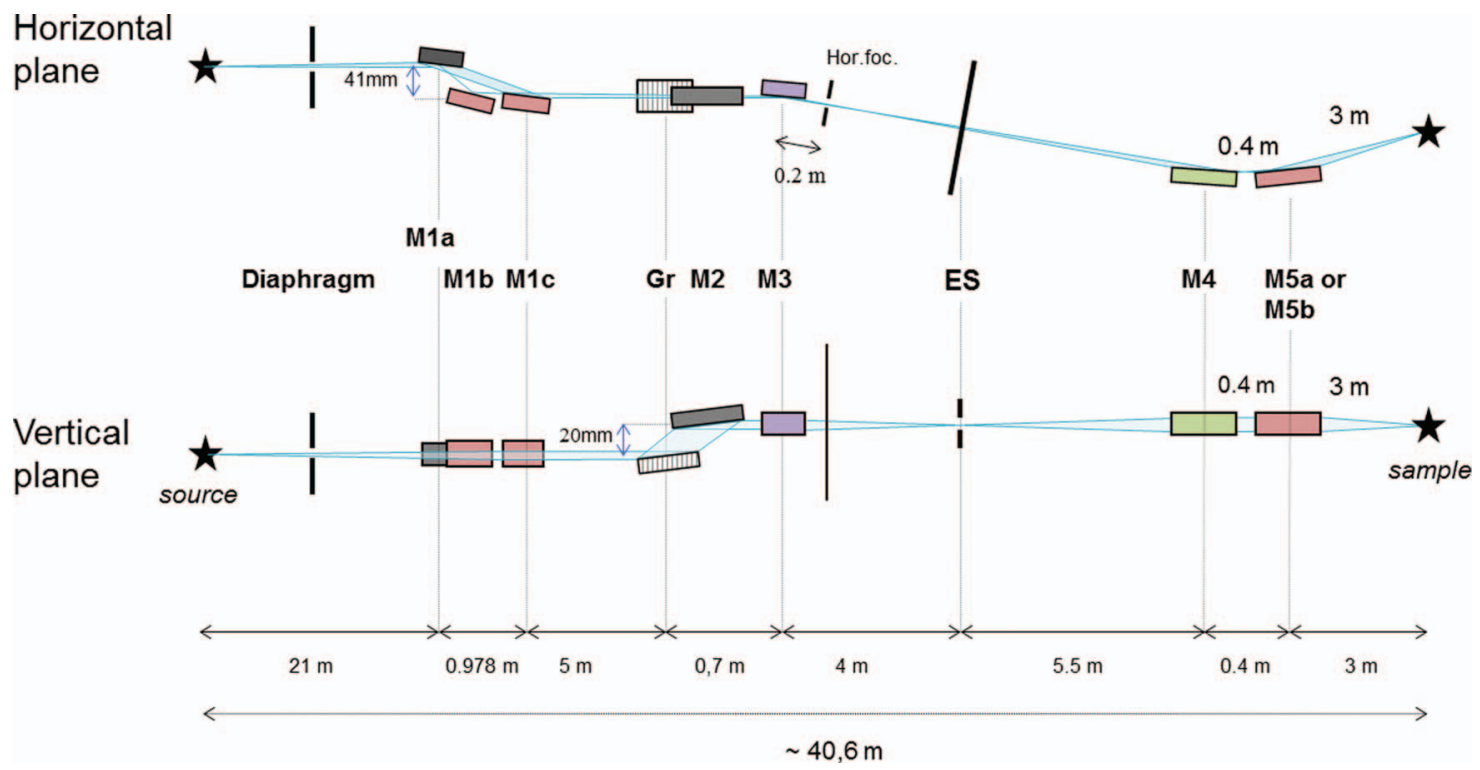

FIG. 1. Horizontal and vertical view of the schematic layout of the DEIMOS beamline from the source (on the left) to the sample (on the right).

\section{A. Sources}

The DEIMOS straight section (I07-m) is equipped with two undulators: an Apple-II HU-52 undulator and an EMPHU-65 (ElectroMagnet/Permanent magnet Helical Undulator) undulator. ${ }^{8}$

\section{1. $H U-52$}

The $52.4 \mathrm{~mm}$ period of the Apple-II undulator ${ }^{9}$ has been chosen in order to reach all polarizations (linear from horizontal to vertical and circular left and right) from $350 \mathrm{eV}$ up to $2500 \mathrm{eV}$. In practice with the lower gap allowed $(15.5 \mathrm{~mm})$ it is possible to reach $\approx 320 \mathrm{eV}$ in the linear vertical geometry and even smaller energy values with linear horizontal and circular polarizations. However, in order to minimize the deformations on the water cooled $\mathrm{SiC}$ flat mirror, the radiated power should be kept below $\sim 160 \mathrm{~W}$ on the first optic. Therefore, only circular polarizations are allowed in the range $260-350 \mathrm{eV}$. A variable diaphragm is located at $13.09 \mathrm{~m}$ from the middle of the straight section and allows selecting the useful part of the polarized light. ${ }^{10}$ With an optimized diaphragm opening, on the first harmonic the circular polarization rate is $99 \%$ while on the second and third harmonic it is around $98 \% .^{10}$ The pink beam emitted from

TABLE I. Undulators parameters.

\begin{tabular}{lcc}
\hline \hline & HU-52 & EMPHU-65 \\
\hline Device type & Apple-II & EMPHU \\
Technology & NdFeB magnets & NdFeB magnets + electromagnet \\
Max. magnetic field & $\mathrm{B}_{\mathrm{x}}=0.53 \mathrm{~T}$ & $\mathrm{~B}_{\mathrm{x}}=0.28 \mathrm{~T}$ \\
& $\mathrm{~B}_{\mathrm{z}}=0.76 \mathrm{~T}$ & $\mathrm{~B}_{\mathrm{z}}=0.24 \mathrm{~T}$ \\
Period length & $52.4 \mathrm{~mm}$ & $65.0 \mathrm{~mm}$ \\
Number of periods & $30+2$ & 24 \\
Minimum gap & $15.5 \mathrm{~mm}$ & $14.7 \mathrm{~mm}$ \\
Phase-shift range & $\pm 26.2 \mathrm{~mm}$ & $\pm 350 \mathrm{~A}$ \\
\hline \hline
\end{tabular}

the undulators can be visualized and characterized using the so-called DiagOn device. ${ }^{11}$ This equipment, developed at SOLEIL, consists of a multilayer and a $\mathrm{Si}(111)$ single crystal, both reflecting upward the beam in Bragg diffraction on a scintillator screen (YAG:Ce). The multilayer and the crystal have been optimized to work for different ranges of undulator gaps according to their diffracted energy. The multilayer is made of 50 pairs of $\operatorname{Cr}(1 \mathrm{~nm}) / \mathrm{Sc}(1.4 \mathrm{~nm})$ layers and reflects the beam at $367.5 \mathrm{eV}$ according to its fixed Bragg angle of $45^{\circ}$; it is optimized to study the first two harmonics of the undulator set for linear horizontal (LH) polarized light. The $\mathrm{Si}(111)$ crystal, which reflects the beam at $2796 \mathrm{eV}$, is used to study the third to the seventeenth undulator harmonics (still with LH polarization). This makes the DiagOn an ideal tool to analyse the HU-52 undulator properties over a wide harmonic range. ${ }^{12}$

\section{EMPHU-65}

The purpose of the EMPHU-65 undulator is to supplement the HU-52 undulator by enhancing sensitivity. ${ }^{13}$ Indeed, the use of an electromagnet to generate the vertical magnetic field allows to switch rapidly (5 $\mathrm{Hz}$ repetition rate) from right to left circular polarization. The drawback of using coils is that emission of linear horizontal polarization is made impossible. Nevertheless, its ability to flip rapidly between circular polarizations makes it an ideal source to measure very small XMCD and XNCD signals. An alternative way of switching rapidly the polarization has been developed on other beamlines. It consists in the use of two identical undulators set at two different polarizations; the two sources are switched at each energy point of the spectrum. ${ }^{14,15}$ This approach implies that the beam follows different optical paths for each polarization, thus potentially leading to nonlinear effects which may jeopardize the measurements, in particular when looking for very small XMCD signals. By using the same optical path, the use of an EMPHU ensures a very high 
TABLE II. Optical parameters.

\begin{tabular}{|c|c|c|c|c|c|c|c|c|c|c|c|}
\hline Parameter & M1a & M1b & M1c & Gra & Grb & M2a & M2b & M3 & M4 & M5a & M5b \\
\hline Shape & Flat & Toroid & Toroid & Flat & Flat & Flat & Flat & Cylinder & Sphere & Toroid & Toroid \\
\hline $\begin{array}{l}\text { Dist. from the } \\
\text { straight section } \\
\text { center }(\mathrm{m})\end{array}$ & 21 & 21.470 & 21.978 & 27.448 & 27.448 & $\sim 27.7^{\mathrm{b}}$ & $\sim 27.7^{\mathrm{b}}$ & 28.148 & 37.648 & 38.048 & 38.048 \\
\hline $\begin{array}{l}\text { Max. absorbed } \\
\text { power }(\mathrm{W})\end{array}$ & $\sim 160$ & $\sim 15$ & $\sim 15$ & $\sim 1$ & $\sim 1$ & $\ldots$ & $\ldots$ & - & - & - & - \\
\hline \multicolumn{12}{|l|}{ Dimension $(\mathrm{mm})$} \\
\hline Length & 220 & 130 & 230 & 70 & 50 & 290 & 200 & 40 & 340 & 320 & 320 \\
\hline Width & 20 & 25 & 25 & 40 & 20 & 30 & 20 & 20 & 35 & 35 & 35 \\
\hline Thick & 25 & 60 & 60 & 60 & 60 & 60 & 60 & 20 & 40 & 40 & 40 \\
\hline $\begin{array}{l}\text { Beam footprint } \\
\left(\mathrm{mm}^{2}\right)\end{array}$ & $184 \times 4$ & $92 \times 4$ & $184 \times 4$ & $60 \times 1$ & $35 \times 1$ & $30 \times 1$ & $30 \times 1$ & $20 \times 10$ & $320 \times 3$ & $300 \times 3$ & $300 \times 3$ \\
\hline $\begin{array}{l}\text { RMS roughness } \\
(\mathrm{nm})^{\mathrm{a}}\end{array}$ & 0.32 & 0.14 & 0.27 & 0.6 & 0.25 & 0.10 & 0.23 & 0.15 & 0.10 & 0.20 & 0.16 \\
\hline $\begin{array}{l}\text { RMS slope error } \\
\tan .(\mu \mathrm{rad})^{\mathrm{a}}\end{array}$ & 0.8 & 2.6 & 2.8 & 0.23 & 0.6 & 0.4 & 0.6 & 1.0 & 1.1 & 1.3 & 0.7 \\
\hline $\begin{array}{l}\text { RMS slope error } \\
\text { sag. }(\mu \mathrm{rad})^{\mathrm{a}}\end{array}$ & 0.4 & 0.7 & 1.91 & $\ldots$ & 0.7 & 0.8 & 1.1 & 2.2 & 3.0 & 4 & 2.5 \\
\hline Bulk material & SiC-CVD & $\mathrm{Si}$ & $\mathrm{Si}$ & $\mathrm{Si}$ & $\mathrm{Si}$ & $\mathrm{Si}$ & $\mathrm{Si}$ & $\mathrm{Si}$ & $\mathrm{SiO}_{2}$ & $\mathrm{SiO}_{2}$ & $\mathrm{SiO}_{2}$ \\
\hline Coating & $\mathrm{Pt}$ & $\mathrm{Pt}$ & $\mathrm{Rh}$ & $\mathrm{Pt}$ & $\begin{array}{l}{\left[\mathrm{B}_{4} \mathrm{C} / \mathrm{Mo}_{2} \mathrm{C}\right]} \\
2 \mathrm{~d}=5.36 \mathrm{~nm}\end{array}$ & $\mathrm{Pt}$ & $\begin{array}{c}{\left[\mathrm{B}_{4} \mathrm{C} / \mathrm{Mo}\right] 2 \mathrm{~d}} \\
=5.35 \mathrm{~nm}\end{array}$ & $\mathrm{Pt}$ & $\mathrm{Pt}$ & $\mathrm{Pt}$ & $\mathrm{Pt}$ \\
\hline $\begin{array}{l}\text { Total deflection } \\
\text { angle }\left({ }^{\circ}\right)\end{array}$ & $5.06^{\circ}$ or $2.4^{\circ}$ & $5.06^{\circ}$ & $2.4^{\circ}$ & Variable & Variable & Variable & Variable & $2.4^{\circ}$ & $2.4^{\circ}$ & $2.4^{\circ}$ & $2.4^{\circ}$ \\
\hline Tangential radius ${ }^{\mathrm{a}}$ & $-19.5 \mathrm{~km}^{\mathrm{c}}$ & $233.03 \mathrm{~m}$ & $455.5 \mathrm{~m}$ & $-3.7 \mathrm{~km}^{\mathrm{c}}$ & $6.7 \mathrm{~km}$ & $360 \mathrm{~km}$ & $42.3 \mathrm{~km}$ & $592.8 \mathrm{~m}$ & $296.2 \mathrm{~m}$ & $955 \mathrm{~m}$ & $2.9 \mathrm{~km}$ \\
\hline Sagittal radius ${ }^{\mathrm{a}}$ & $3.39 \mathrm{~km}$ & $1894 \mathrm{~mm}$ & $924 \mathrm{~mm}$ & $\ldots$ & $2.8 \mathrm{~km}$ & $1.7 \mathrm{~km}$ & $1.8 \mathrm{~km}$ & $167.8 \mathrm{~mm}$ & $188.1 \mathrm{~m}$ & $83.6 \mathrm{~mm}$ & $150.3 \mathrm{~mm}$ \\
\hline $\begin{array}{l}\text { Line density } \\
(1 / \mathrm{mm})\end{array}$ & $\ldots$ & $\ldots$ & $\ldots$ & 1600 & 2400 & $\ldots$ & $\ldots$ & - & - & - & - \\
\hline $\begin{array}{l}\text { Groove depth } \\
(\mathrm{nm})^{\mathrm{a}}\end{array}$ & $\ldots$ & $\ldots$ & $\ldots$ & $\begin{array}{l}3.6 \text { to } 15.4 \\
\text { on } 28 \mathrm{~mm}\end{array}$ & $\approx 2.48$ & $\ldots$ & $\ldots$ & - & - & - & - \\
\hline $\begin{array}{l}\text { Groove width to } \\
\text { period ratio }\end{array}$ & $\ldots$ & $\ldots$ & $\ldots$ & $0.43-0.56$ & 0.46 & $\ldots$ & $\ldots$ & - & - & - & - \\
\hline
\end{tabular}

${ }^{\mathrm{a}}$ Measured values.

${ }^{\mathrm{b}}$ Varies with the energy and the factor $c$.

${ }^{\mathrm{c}} \mathrm{A}$ minus sign denotes a convex surface.

reproducibility and stability of the beam and consequently an improved sensitivity in the dichroic measurements (see Ref. 16 for some examples of dichroism in absorption experiments performed at the very edge of this technique using an EMPHU undulator).

\section{B. M1 mirrors chamber}

The first optical chamber allows choosing between two optical paths, M1a-M1b or M1a-M1c, by translating in and out of the optical path the M1b mirror. The mirrors M1b and M1c differ by their coatings, platinum, and rhodium, respectively. The M1a-M1b path is optimized for high energy harmonics rejection below $1300 \mathrm{eV}$ due to a working angle of $2.53^{\circ}$, whereas M1a-M1c is used for higher energies (working angle on the Rh coated M1c mirror: $1.20^{\circ}$ ) (see the cross curve on Figure 4(a)). For the maximum angular acceptance of $0.2 \times 0.2 \mathrm{mrad}^{2}$ defined by the variable diaphragm, the footprints on M1a are $92 \times 4 \mathrm{~mm}^{2}$ and 184 $\times 4 \mathrm{~mm}^{2}$ for respectively the M1a-M1b and M1a-M1c trajectories. The power coming on the first optics (maximum of $160 \mathrm{~W}$ for an angular acceptance of $0.2 \times 0.2 \mathrm{mrad}^{2}$ according to the calculation using $\mathrm{SRW}^{17}$ ) is dissipated using a water cooled SiC-CVD mirror (SiC-CVD from Rohm and Haas having a thermal conductivity of $300 \mathrm{~W} / \mathrm{mK}$ ). The deformation due to the thermal heat load has been evaluated by finite element analysis and input to the optical ray tracing. ${ }^{18}$ The comparison of these calculations to the perfectly flat mirror case show that, at worse, the resolving power is reduced by $\sim 6 \%$ and the horizontal beam size is increased by a factor $1.3 \pm 0.1$.

The horizontal deflecting toroidal mirror (M1b or M1c) focus this light in the horizontal plane to a point located $200 \mathrm{~mm}$ after the M3 mirror (inside the monochromator chamber) while in the vertical direction the beam is collimated in an almost parallel beam.

\section{Plane grating monochromator (PGM)}

The monochromator is equipped with two plane gratings, Gra and Grb, which are respectively a variable groove depth $(\text { VGD })^{19}$ grating with 1600 lines $/ \mathrm{mm}$, and a $\mathrm{Mo}_{2} \mathrm{C} / \mathrm{B}_{4} \mathrm{C}$ alternate multilayer (AML) grating with 2400 lines $/ \mathrm{mm} .{ }^{20-22}$ The VGD grating is optimized for the energy range $240-1500 \mathrm{eV}$ while the AML grating is optimized for the energy domain $1000-2500 \mathrm{eV}$. The efficiency of both gratings is detailed in Sec. III C. The dispersed light is vertically deflected onto the associated M2 plane mirror (M2a or M2b in Table II); note 
that in the case of the AML grating, M2b is a multilayer mirror with a matched period. The beam is then deviated in the horizontal plane by a cylinder mirror M3 which focuses the beam on the exit slit (see Fig. 1).

With the VGD grating the monochromator is usually operated in the Petersen geometry with a fixed $c$ factor. ${ }^{23}$ This $c$ factor is defined as: $c=\sin \beta / \sin \alpha$, where $\alpha$ is the grazing incidence angle of the light on the grating and $\beta$ the grazing diffraction angle. The monochromator is working in the first outside diffraction order, with the grating as the first optics; hence the $c$ values are below 1. Usable $c$ factor values are comprised between 0.05 and 0.4 ; the smaller $c$ values correspond to the higher dispersion, i.e., to the higher resolving power for a given exit slit (and consequently a lower transmitted flux). The high groove density of the VGD grating (1600 lines/mm) has been chosen to improve the energy stability. As a matter of fact, achieving a given energy resolution with a large grating dispersion and a moderately closed exit slit reduces the influence of the mechanical angular accuracy (of both the grating and the mirror) onto the energy accuracy. Nevertheless, it is also possible to achieve large resolving power if one decides to work with a closed exit slit, and the ability of changing the $c$ factor gives further flexibility.

In order to determine the resolving power of the beamline, resolution measurements have been performed using a gas cell located between the exit slit and the Wolter chamber ${ }^{24}$ (mirror pair M4-M5). In this cell a stable pressure (around $1 \times 10^{-3}$ mbar) is established with $\mathrm{N}_{2}$ or Ne gas. An electrode inside this chamber collects the electrons emitted during the photon absorption; the current recorded gives a good measurement of the x-ray absorption spectroscopy (XAS). On Figure 2, we plot a typical $K$-edge XA spectrum for $\mathrm{N}_{2}$.

From such XA spectrum, using a Lorentzian function decomposition, we can determine the variations of the full width at half maximum as a function of the $c$ factor and the opening of the exit slits. The use of the $K$-edge of $\mathrm{N}_{2}$ or $\mathrm{Ne}$ allows characterizing this dependence at respectively 410 and $870 \mathrm{eV}$. Using this method, and knowing the natural width of

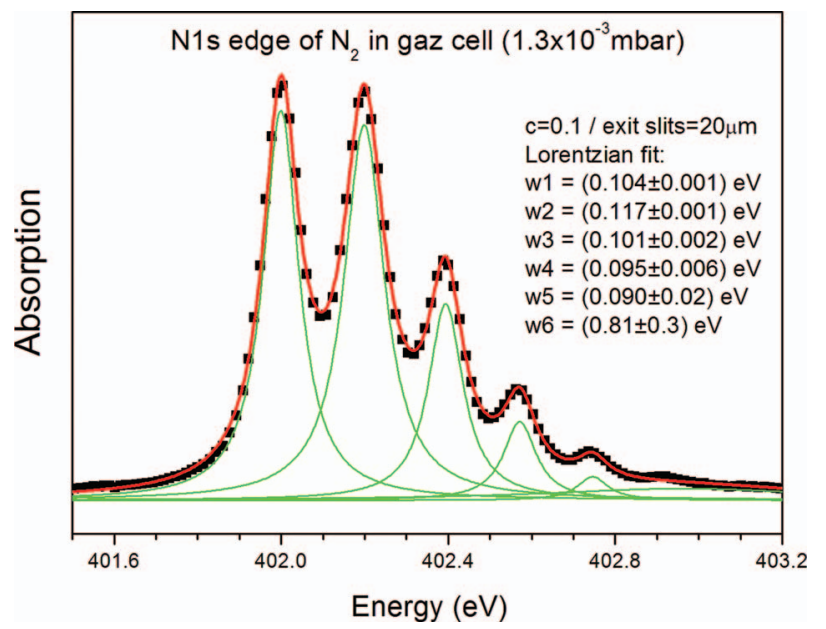

FIG. 2. K-edge XA spectrum for N2 with c set to 0.1 and the exit slits open at $20 \mu \mathrm{m}$; dotted curve: experimental data, red curve: total spectrum of 6 Lorentzians, and green curves: individual Lorentzian contributions. the K-edges (i.e., $0.123 \mathrm{eV}$ and $0.230 \mathrm{eV}$ for respectively $\mathrm{N}_{2}$ and $\mathrm{Ne}^{25}$ ), we verified that the resolving power of the VGD grating is within $8 \%$ of the theoretical value, i.e., an ultimate resolving power between 30000 and 35000 for the energy range $350-1500 \mathrm{eV}$ and for a factor $c$ of 0.2 .

The energy stability was measured using a reference $\mathrm{Fe}_{3} \mathrm{O}_{4}$ single crystal sample, which is chemically very stable under x-rays and provides very sharp $L_{2,3}$ edges mandatory for such measurement. The principle consists in recording over several hours the same spectrum in exactly the same conditions (light polarization and sample state). Then, spectra taken at different moments (separated by $1-2 \mathrm{~h}$ ) are subtracted to each other and the resulting signal (ideally 0 ) is compared to the mathematical derivative of the average of all measurements. Using this method, we could measure a stability in energy of $\approx 1 \mathrm{meV} / \mathrm{h}$, which is a very competitive value. This value is comparable to the drift in energy (read from the encoders' rotations of the monochromator) due to the variations in temperature produced by the day-night cycles, although the monochromator has its own hutch with a dedicated air-conditioner which keeps the temperature variations below $\pm 0.5^{\circ} \mathrm{C}$. This means that the energy stability is mainly limited, in our case, by the thermal stability of the monochromator. It should nevertheless be noted that the monochromator does not have an entrance slit to define the source, but rather uses the image of the undulator source reflected by the M1 mirror pair. This means that the energy stability can also be affected by temperature variation of the first optics hutch and machine stability.

\section{Wolter mirror pair}

The last optical stage is a simplified Wolter mirror combination associating a spherical mirror M4 and a toroidal mirror M5 as approximations of the ellipsoidal shapes of the canonical Wolter combination. ${ }^{24}$ The two successive reflections in the horizontal plane are used to cancel the coma aberration in this plane, and hence accommodate the large image aperture which results from the large horizontal demagnification imposed by SOLEIL horizontal source size. Two toroidal mirrors (M5a and M5b) are available and can be switched under vacuum in order to produce two different beam sizes in the end-station located $3 \mathrm{~m}$ downstream. While M5a exactly focuses on the experimental point, M5b is focusing somewhat beyond in order to increase by a factor of $\sim 10$ the spot size on the sample, both horizontally and vertically. This divides by $\sim 100$ the photon density on the sample going from a spot size of $\sim 80 \times 80 \mu \mathrm{m}^{2}$ for M5a to $\sim 800 \times 800 \mu \mathrm{m}^{2}$ for M5b. Additionally, due to its angle tolerant design, the Wolter permits to move the beam on the sample in a range of a few millimeters.

\section{E. End-station}

The end-station consists of a superconducting magnet providing $\pm 7 \mathrm{~T}$ along the x-rays beam or $\pm 2 \mathrm{~T}$ perpendicular to the beam. The variable temperature insert (VTI) has been developed for temperatures in the $1.5-370 \mathrm{~K}$ range. ${ }^{7}$ The absorption signal can be recorded in 3 different ways: (i) in 
transmission mode (for semi-transparent samples) with a removable diode ${ }^{26}$ located at the back of the end-station chamber, (ii) in total electron yield, and (iii) in fluorescence yield. For all these modes, the currents are measured with Novelec electrometers connected to a counting card NI-6602 from $\mathrm{Na}$ tional Instruments. ${ }^{27}$

The end-station is connected to a glove-box and to a set of ultra-high vacuum (UHV, $<10^{-9}$ mbar) chambers dedicated to in situ sample conditioning, including sample preparation and characterization under inert atmosphere (standard wet chemistry techniques) and under UHV conditions (evaporation, $\mathrm{Ar}^{+}$sputtering, annealing, Auger electron spectroscopy, low energy electron diffraction, and scanning tunneling microscopy).

\section{SPECIFIC DEVELOPMENTS}

\section{A. Turboscan (on-the-fly energy scan)}

As mentioned above, the DEIMOS beamline has been optimized for dichroic measurements obtained by comparison of XA spectra recorded with different polarizations and/or different magnetic/electronic states. This statement implies that for such measurements a very good stability and fast acquisition process are necessary in order to minimize the impact of the residual instabilities due to the experimental environment. This fast data acquisition is a common issue on beamlines performing scans in energy. In a conventional step-bystep approach, the monochromator and the insertion device are sequentially driven to the desired photon energies. The limiting factor of this approach is the time needed to accurately position all the motors: gratings and mirrors cradles in the monochromator as well as gap and phase motors of the insertion device, as their acceleration and deceleration are time consuming.

An alternative approach is to use an on-the-fly energy scanning mode. However, rigorous approach requires sophisticated multi-axis motion control with nonlinear motion trajectories; this is still part of an on-going development at SOLEIL. In the meantime, we have thus chosen to rely on a software based solution ${ }^{28}$ using the available Tango tools (the so-called Tango is an object oriented distributed control system used at SOLEIL ${ }^{29}$ ). It consists in a software synchronization of the monochromator and the undulator motors by determining proper initial position, final position, and speed for each motor.

From the user point of view, the parameters are the start $\left(E_{S}\right)$ and final $\left(E_{F}\right)$ energies, the total time $(t)$ and the integration time $(d t)$. Using these values $\left(E_{S}, E_{F}\right.$, and $\left.t\right)$, the trajectories (initial, final positions, and speed) of the 2 rotations of the monochromator and the gap of the undulator are determined. When the on-the-fly scan is launched all these motor positions are recorded by a counting card in a synchronous fashion with the integration time $d t$. The trajectory in energy is then reconstructed from these known motor positions. To improve the synchronization between the energy of the monochromator and the energy of the undulator, a master-slave dynamical coupling is established between the two devices. In practice, as the photon energy on the sample is always given by the monochromator, the latter is given the role of "master." During the scan, every $500 \mathrm{~ms}$, the position in energy of the insertion device is compared to the position of the monochromator and its speed is adjusted in order to minimize this offset.

Compared to a step-by-step XA spectrum, this on-the-fly spectrum allows a gain of $\approx 10$ in time with similar statistics and resolution in energy. In addition, we have checked several important points of these on-the-fly scans: (i) $c$ factor: as explained previously, the monochromator is normally operated with a fixed c factor. But during the on-the-fly scan, the coupling between grating and M2 mirror rotations is not ensured. Nevertheless, we can calculate at the end of a scan the actual $\mathrm{c}$ factor from the $\alpha$ and $\beta$ angle values recorded at each measurement point, and it appears that it never deviates by more than $0.5 \%$ from the desired value. This deviation is low enough to claim that acquisition conditions are similar to a step-by-step scan. (ii) Monochromator-undulator synchronization: the feedback during the scan allows reducing the offset between the monochromator and undulator to values as low as $3 \mathrm{eV}$. Compared to the typical width of the undulator peak $(15-40 \mathrm{eV})$, we can conclude that the synchronization of the undulator and the monochromator is very good, even for a wide scan of several hundreds of $\mathrm{eV}$. (iii) Polarization: the phase is not adjusted during the on-the-fly scan. In the case of circular polarized light, a constant phase over an energy range of $\sim 100 \mathrm{eV}$ means a loss in the polarization rate limited to few percents. Such deviation is well below the typical errors bars on a dichroic measurement.

In conclusion, the on-the-fly scan in energy is a powerful tool to perform XA spectra in a fast and accurate way. The typical time to acquire a scan of $\sim 100 \mathrm{eV}$ width is $2-3 \mathrm{~min}$.

\section{B. EMPHU-65}

Another elegant way to perform dichroic measurements in absorption is to record at each point in energy the dichroic signal. In the case of XMCD, this is obtained by flipping the circularly polarized light direction at each point in energy. To perform such measurements, we have chosen a second undulator which uses a combination of permanent magnets and electromagnets. The electromagnets allow flipping the circular polarization at a rate of $5 \mathrm{~Hz}$. Practically, this means that at each energy point of an absorption spectrum the signal is measured for the two circular polarizations which are switched within $0.2 \mathrm{~s}$. This very short delay between the two measurements minimizes the impact of all the measurement instabilities and offers the unique capability to measure very weak dichroic signals.

While tuning such a device the beam steering in the undulator is a critical parameter, and should be identical when switching from one polarization state to the other. This has been monitored using the diaphragm as a slit to image the beam intensity profile, and it allowed to define the fine parameters of the insertion device (correction tables). As a final check, the beam on the sample $\left(\sim 80 \times 80 \mu \mathrm{m}^{2}\right)$ has been imaged on a YAG:Ce screen and no change in position has been observed when flipping the polarization, within the resolution of our measurement $(10 \mu \mathrm{m})$. 


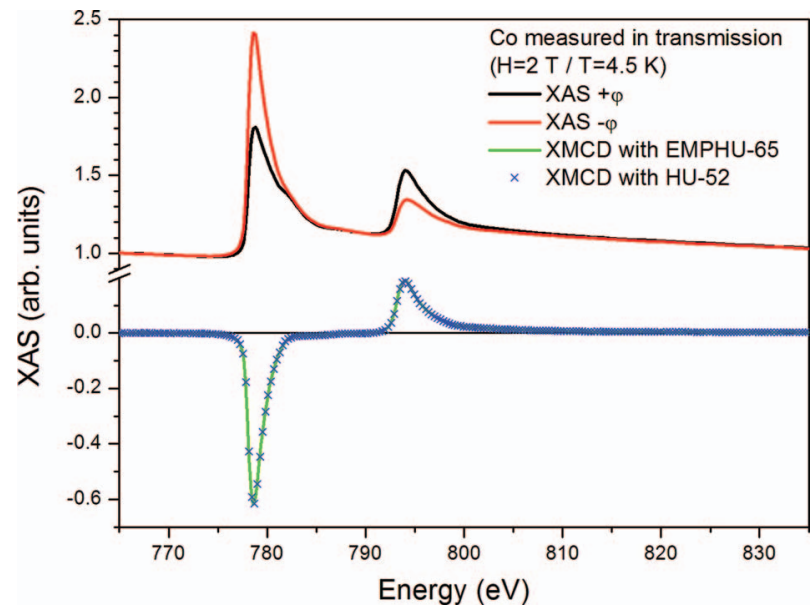

FIG. 3. XAS and XMCD at the $L_{2,3}$ edges of Co. The solid lines correspond to the measurements obtained using the EMPHU-65 while the doted XMCD spectrum is given as a reference and has been obtained using the HU-52 in the same conditions.

A second important issue is the polarization symmetry between circular left and right. The calculations using the magnetic measurements predicted polarization rates of $99.99 \%$ and $99.82 \%$ for respectively the right and left circulars polarized light (in the case of a gap of $27.5 \mathrm{~mm}$ corresponding to $\sim 800 \mathrm{eV}$ ). The first XMCD spectra obtained on a Co sample confirm this statement: the deduced circular polarization rate indicates a circular polarization rate above $99 \%$ (Figure 3). Indeed, the XMCD signal has exactly the same amplitude as the one obtained with the HU-52, leading to the magnetic moment expected from the sum rules. These results confirm the good performance of the EMPHU-65.

\section{Multilayer grating}

The AML grating has been developed to improve significantly the efficiency of the monochromator at high energies. Lamellar gratings have rather low efficiencies at energies above $\sim 1500 \mathrm{eV}$ and DEIMOS VGD grating is not optimized to work at energies higher than $\sim 1200 \mathrm{eV}$. Blazed gratings with triangular line profiles are a known way of overcoming this limitation: ${ }^{30}$ they offer high efficiency in the $2 \mathrm{keV}$ energy range when the blaze angle (angle of the triangular facet with the grating plane) is in the order of $1-2^{\circ}$, and they can be coupled with a grazing incidence mirror to offer a good throughput. Nevertheless, the shallow angle of the profile and the manufacturing precision it requires make the realization of such gratings difficult to master on a large optical surface. Moreover, they are not suitable for holographic fabrication and generally rely on a mechanical ruling with all associated regularity issues. For these reasons SOLEIL, together with Horiba Scientific (formerly Horiba Jobin-Yvon SAS), have been working in the last years on an alternative technology named AML grating. ${ }^{31}$ The basic idea is to deposit alternate layers of two materials on a lamellar grating substrate in order to synthesize a $2 \mathrm{D}$ periodic structure in the plane perpendicular to the grating lines. The AML grating has properties similar to crystals used in high energy double crystal monochromator, namely only waves satisfying a Bragg condition are

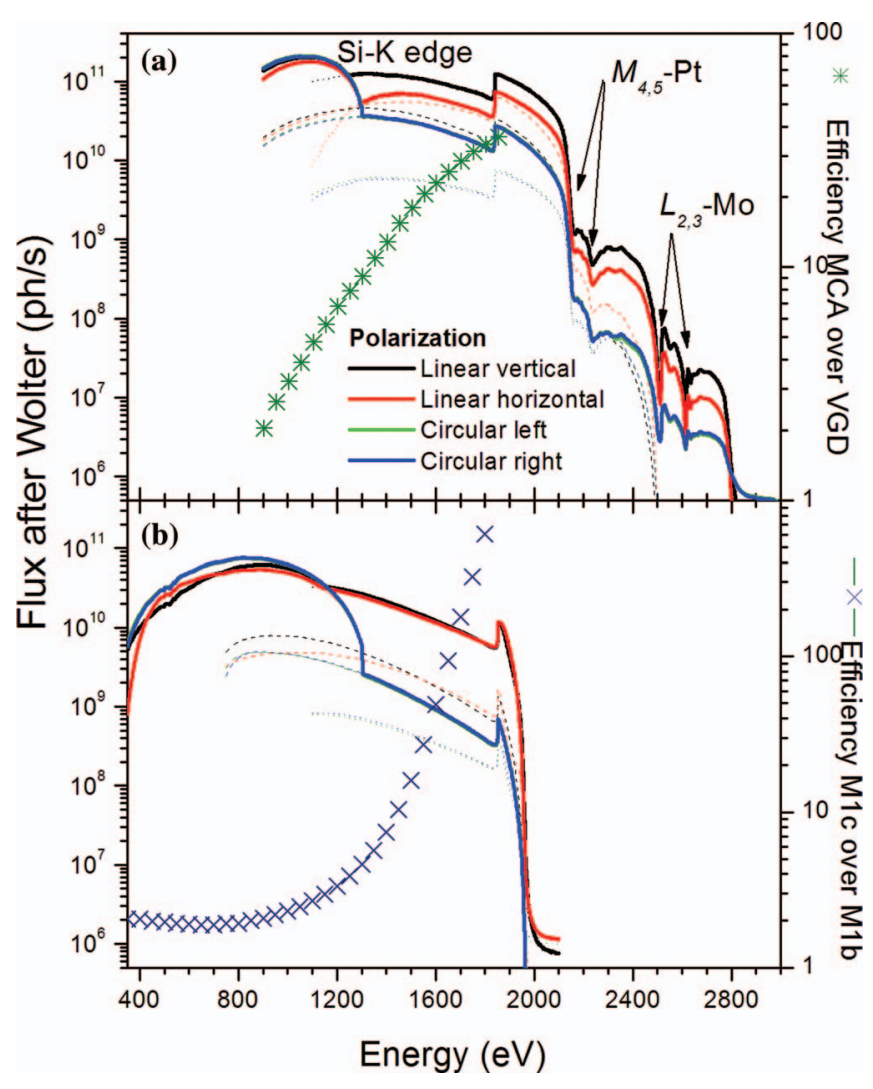

FIG. 4. Flux measured after the Wolter chamber for different light polarizations and a ring current of $430 \mathrm{~mA}$. (a) Using the AML grating (exit slit set to $200 \mu \mathrm{m}$ ) and (b) the VGD grating (exit slit set to $100 \mu \mathrm{m}$ ). The 1st harmonic goes up to $\sim 1300 \mathrm{eV}$ and the 2 nd (dashed lines) and 3rd harmonics (dotted lines) are used to go to higher energies value. The symbols $-x-$ in blue (b) represent the efficiency of the M1a-M1c optical path over M1a-M1b while the green -*- (a) illustrate the efficiency of the AML grating over the VGD. The Si- $K$ edge is due to the material of the diode while the Mo- $L_{2,3}$ and Pt- $M_{4,5}$ edges correspond to the optical coating materials.

reflected. This enhances the diffraction efficiency in the allowed orders. In a monochromator, the AML grating should be paired with a mirror coated with a similar multilayer which compensates the grating deviation. ${ }^{20}$ DEIMOS AML grating and multilayer mirror pair is the first to be fully commissioned for operation at SOLEIL.

DEIMOS AML grating Grb was fabricated by deposition of 30 periods of a $\left[\mathrm{Mo}_{2} \mathrm{C} / \mathrm{B}_{4} \mathrm{C}\right]$ multilayer coating, on a silicon laminar grating substrate etched with a line density of $2400 \mathrm{~mm}^{-1}$. The materials were chosen for allowing high reflectivity around $2 \mathrm{keV}$ and their ability to form clean interfaces, almost without any interdiffusion. The layer period, $5.36 \mathrm{~nm}$, was selected to match the grating groove depth and results in a Bragg angle of about $0.69^{\circ}$. This grating/mirror pair has an optimal efficiency before Mo- $L$ edges in the 1000$2450 \mathrm{eV}$ range. The details of the fabrication and the choice of materials for the AML, as well as the first characterization, are given elsewhere (see Refs. 20 and 21) and we will concentrate in this paper on the gain in terms of photon flux.

The flux was measured, using a calibrated AXUV-100 photodiode, ${ }^{32}$ for the 2 optical pathways M1a-M1b and M1aM1c in the case of the classical VGD grating and with the AML grating. In both cases, the aperture was $0.1 \times 0.1 \mathrm{mrad}$ 


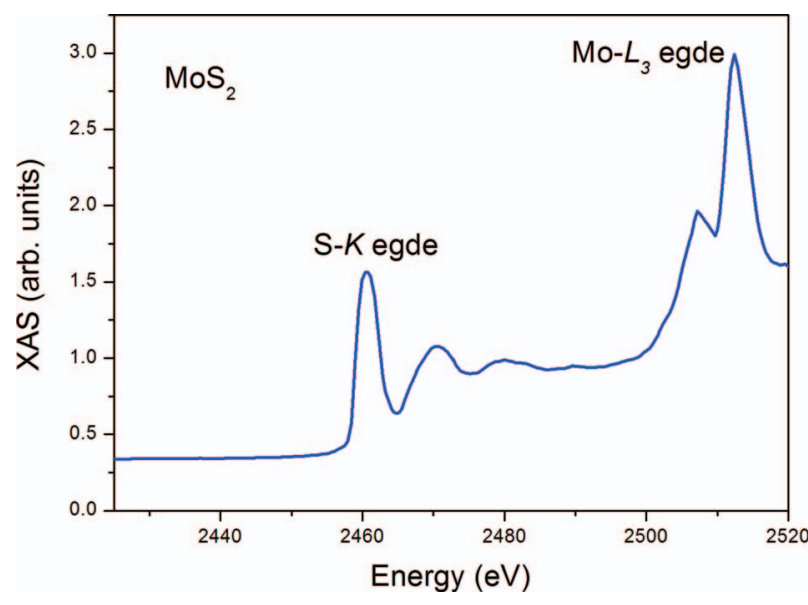

FIG. 5. X-ray absorption spectrum of S- $K$ and Mo- $L_{3}$ edges for $\mathrm{MoS}_{2}$.

and only the settings of the monochromator were different: for the VGD the $\mathrm{c}$ factor was set to 0.2 and the exit slit to $100 \mu \mathrm{m}$, to be close to the usual settings, while for the AML grating the exit slit was set to $200 \mu \mathrm{m}$ to improve the flux at the cost of the ultimate resolution. Flux measurements have been done for all 4 standard light polarizations produced by the HU-52, using the first three harmonics to cover an energy range from 350 to $3000 \mathrm{eV}$ (see Figure 4).

On the same figure, we also represent different efficiencies (plot with the large symbols): the efficiency of the M1aM1c optical path over M1a-M1b and more interestingly the AML grating efficiency over the VGD. From this curve, we see that the AML grating offers a good efficiency from $\sim 1000 \mathrm{eV}$ and that above $2000 \mathrm{eV}$ it is the only way to get some flux on the sample. Although there is a strong flux drop above $2000 \mathrm{eV}$, due to the Pt and Mo mirrors coatings, the AML grating still provides a reasonable flux and we are able to perform absorption measurements at this energy as can be seen from the x-ray absorption spectrum of $\mathrm{MoS}_{2}$ recorded at $\mathrm{S}-K$ and Mo- $L_{3}$ edges displayed in Figure 5.

\section{CONCLUSION AND PROSPECTS}

The DEIMOS beamline has been optimized for XAS and all types of dichroic measurements in the $350-2500 \mathrm{eV}$ energy range. In order to reach such high energy on a soft Xray beamline, we developed AML gratings. The capability to record very small XMCD signal has been improved by the implantation of the EMPHU insertion device, first hybrid electromagnet/permanent magnet undulator build to work in the soft energy range. Other instrumental developments are in progress: (i) additionally to the standard VTI we are developing an insert with 12 current leads to the sample. These current leads can have several purposes, such as, for instance, the application of an electrical potential difference across the sample, or the possibility to perform 4-points resistance measurements simultaneously to XMCD measurements. (ii) $\mathrm{We}$ have started the development of a third dedicated VTI for measurements from 200 to $1000 \mathrm{mK}$ using a ${ }^{3} \mathrm{He}-{ }^{4} \mathrm{He}$ dilution refrigerator. (iii) We will install by the end of the year a new end-station consisting of an electromagnet with a VTI dedicated to measure in the high temperature range from 20 to $1000 \mathrm{~K}$. (iv) With the growing interest of time resolved magnetic studies we are now considering the possible development of a second branch line dedicated to time resolved measurements in the $0.1 \mathrm{ps}$ scale using the sliced beam. ${ }^{33}$ This branch would be optimized for providing the maximum flux on the sample knowing that the slicing scheme means the source flux will be reduced by 6 orders of magnitude.

\section{ACKNOWLEDGMENTS}

The authors would like to acknowledge all the staff from SOLEIL who have made possible the building of the DEIMOS beamline. We specially acknowledge the contribution of M. Thomasset and S. Brochet whose results of optics metrology are reported in Table II. We are grateful to the Institut de Physique et Chimie de Strasbourg (IPCMS) for their support during the building and the commissioning. Thanks are due to F. Delmotte, E. Meltchakov, and F. Bridou from Institut d'Optique Graduate School (IOGS) for multilayer deposition and $\mathrm{Cu}-\mathrm{K}_{\alpha}$ characterization. The preparation chambers have been partially funded by the Agence National de la Recherche (Grant No. ANR-05-NANO-073). The gloves-box, provided by the Institut de Minéralogie et de Physique des Milieux Condensés (IMPMC), has been funded by the Agence National de la Recherche (Grant No. ANR-07BLANC-0275). The cryomagnet has been partially funded by the IPCMS and IMPMC.

${ }^{1}$ S. Sasaki, K. Miyata, and T. Takada, Jpn. J. Appl. Phys. 31, L1794 (1992); S. Sasaki, T. Shimada, K. Yanagida, H. Kobayashi, and Y. Miyahara, Nucl. Instrum. Methods A 347, 87 (1994).

${ }^{2}$ A. Rogalev, J. Goulon, G. Benayoun, P. Elleaume, J. Chavanne, C. Penel, and P. Van Vaerenbergh, Proc. SPIE 3773, 275 (1999); P. Elleaume, CAS, Brunnen, 2-9 July 2003.

${ }^{3}$ F. Djeghloul, F. Ibrahim, M. Cantoni, M. Bowen, L. Joly, S. Boukari, P. Ohresser, F. Bertran, P. Le Fevre, P. Thakur, F. Scheurer, T. Miyamachi, R. Mattana, P. Seneor, A. Jaafar, C. Rinaldi, S. Javaid, J. Arabski, J. P. Kappler, W. Wulfhekel, N. B. Brookes, R. Bertacco, A. Taleb-Ibrahimi, M. Alouani, E. Beaurepaire, and W. Weber, Sci. Rep. 3, 1272 (2013).

${ }^{4}$ P. Gambardella et al., Phys. Rev. Lett. 93, 077203 (2004); Nature (London) 416, 301 (2002).

${ }^{5}$ P. Ohresser et al., Phys. Rev. Lett. 95, 195901 (2005).

${ }^{6}$ B. T. Thole et al., Phys. Rev. Lett. 68, 1943 (1992); P. Carra et al., ibid. 70, 694 (1993).

${ }^{7}$ The temperature on the sample was measured during the commissioning phase directly with a calibrated Si diode glued at the sample place. We have measured temperatures as low as $1.45 \mathrm{~K}$.

${ }^{8}$ J. Chavanne, P. Elleaume, and P. VanVaerenbergh, in Proceedings of EPAC98, Stockholm, Sweden (IOP Publishing, 1998), pp. 317-319 (available online at http://accelconf.web.cern.ch/AccelConf/e98/PAPERS/ TUOB02A.PDF).

${ }^{9}$ F. Marteau, C. Benabderrahmane, P. Berteaud, F. Briquez, P. Brunelle, L. Chapuis, M.-E. Couprie, T. ElAjjouri, J.-M. Filhol, C. Kitegi, O. Marcouille, M. Massal, A. Nadji, L. S. Nadolski, R. Nagaoka, K. Tavakoli, M. Valleau, and J. Veteran, "Development and installation of insertion devices SOLEIL," in Proceedings of PAC09, Vancouver, Canada, 2009 (available online at http://epaper.kek.jp/PAC2009/papers/we5rfp080.pdf).

${ }^{10}$ P. Ohresser, E. Otero, F. Choueikani, S. Stanescu, F. Deschamps, L. Ibis, T. Moreno, B. Lagarde, F. Marteau, F. Scheurer, L. Joly, J. P. Kappler, B. Muller, and Ph. Sainctavit, J. Phys.: Conf. Ser. 425, 212007 (SRI2012) (2013).

${ }^{11}$ K. Desjardins, S. Hustache, F. Polack, T. Moreno, M. Idir, J.-M. Dubuisson, J.-P. Daguerre, J.-L. Giorgetta, S. Thoraud, F. Delmotte, and M.-F. RavetKrill, AIP Conf. Proc. 879, 1101-1104 (2007). 
${ }^{12}$ T. Moreno, E. Otero, and P. Ohresser, J. Synchrotron Radiat. 19(2), 179184 (2012); T. Moreno, E. Otero, X. Dong, and P. Ohresser, Proc. SPIE 8141, 81410H (2011).

${ }^{13}$ F. Marteau, P. Berteaud, F. Bouvet, L. Chapuis, M. E. Couprie, J. P. Daguerre, J. M. Filhol, A. Mary, and K. Tavakoli, "Development of an electromagnetic of an electromagnetic/permanent magnet helical undulator for fast polarisation switching," in Proceedings of IPAC2011, San Sebastián, Spain, 2011 (available online at http://accelconf.web.cern.ch/AccelConf/IPAC2011/papers/thpc151.pdf).

${ }^{14}$ M. R. Weiss, R. Follath, K. J. S. Sawhney, F. Senf, J. Bahrdt, W. Frentrup, A. Gaupp, S. Sasaki, M. Scheer, H.-C. Mertins, D. Abramsohn, F. Schäfers, W. Kuch, and W. Mahler, Nucl. Instrum. Methods Phys. Res., Sect. A 467468, 449-452 (2001).

${ }^{15}$ U. Flechsig, F. Nolting, A. Fraile Rodríguez, J. Krempaský, C. Quitmann, T. Schmidt, S. Spielmann, and D. Zimoch, "Performance measurements at the SLS SIM beamline," AIP Conf. Proc. 1234, 319 (2010).

${ }^{16}$ A. Rogalev, J. Goulon, F. Wilhelm, and A. Bosak, in Magnetism and Synchtrotron Radiation: New Trends, Springer Proceedings in Physics, edited by E. Beaurepaire et al. (Springer, 2010), Chap. 6, p. 133.

${ }^{17} \mathrm{O}$. Chubar and P. Elleaume, in Proceedings of EPAC98, Stockholm, Sweden (IOP Publishing, 1998), pp. 1177-1179 (available online at http://accelconf.web.cern.ch/AccelConf/e98/PAPERS/THP01G.PDF).

${ }^{18}$ T. Moreno and M. Idir, J. Phys. IV Fr. 11, Pr2-527-Pr2-531 (2001).

${ }^{19}$ F. Polack, B. Lagarde, M. Idir, A. Liard, and E. Jourdain, AIP Conf. Proc. 879, 639-642 (2007).

${ }^{20}$ F. Polack, B. Lagarde, M. Idir, A. Liard, E. Jourdain, M. Roulliay, A. Delmotte, J. Gautier, and M. F. Ravet-Krill, AIP Conf. Proc. 879, 489-492 (2007).

${ }^{21}$ F. Choueikani, F. Bridou, B. Lagarde, E. Meltchakov, F. Polack, P. Mercere, and F. Delmotte, "X-ray properties and interface study of B4C/Mo and B4C/Mo2C periodic multilayers," Appl. Phys. A: Mater. Sci. Proc. 111, 191 (2013).

${ }^{22}$ B. Lagarde, F. Choueikani, B. Capitanio, P. Ohresser, E. Meltchakov, F. Delmotte, M. Krumrey, and F. Polack, "High efficiency multilayer gratings for monochromators in the energy range from $500 \mathrm{eV}$ to $2500 \mathrm{eV}$," J. Phys.: Conf. Ser. 425, 152012 (SRI2012) (2013).

${ }^{23}$ H. Petersen, C. Jung, C. Hellwig, W. B. Peatman, and W. Gudat, Rev. Sci. Instrum. 66, 1-14 (1995).

${ }^{24} \mathrm{Ph}$. Troussel, P. Munsch, and J. J. Fermé, Proc. SPIE 3773, 60 (1999); P. Troussel, B. Meyer, R. Reverdin, B. Angelier, G. Lidove, P. Salvatore, and A. Richard, Rev. Sci. Instrum. 76, 063707 (2005).

${ }^{25}$ A. P. Hitchcock and C. R. Brion, J. Electron Spectrosc. Relat. Phenom. 18, 1-21 (1980).

${ }^{26}$ Produced by IRD under the reference SXUV100EUT.

${ }^{27}$ See http://sine.ni.com/nips/cds/view/p/lang/en/nid/1124 for the detailed characteristics of the NI PXI-6602 counting card.

${ }^{28}$ L. Joly, E. Otero, F. Choueikani, F. Marteau, L. Chapuis, and P. Ohresser "Fast continuous energy scan with dynamic coupling of the monochromator and undulator at the DEIMOS beamline," J. Synchrotron Radiat. (submitted).

${ }^{29}$ J.-M. Chaize, A. Götz, W-D. Klotz, J. Meyer, M. Perez, and E. Taure, in Proceedings of ICALEPCS 2001, San Jose, California (IOP Publishing, 2001), pp. 83-85 (available online at http:// accelconf.web.cern.ch/AccelConf/ica01/papers/TUAP004.pdf) (see also eprint arXiv:cs/0111028); see http://www.tango-controls.org.

${ }^{30}$ A. Bianco, G. Sostero, B. Nelles, K. F. Heidemann, and K. F. D. Cocco, "A plane grating with single-layer coating for the sub-nanometer wavelength range," SPIE Proc. 5918, 591810 (2005).

${ }^{31}$ F. Polack, M. Idir, E. Jourdain, and A. Liard, "Réseau de diffraction à empilements multicouches alternés et son procédé de fabrication et dispositifs spectroscopiques comportant ces réseaux," Jobin-Yvon SAS and Synchrotron SOLEIL, patent nos. FR2864252-A1; WO2005064364-A1; US2005270647-A1; EP1700141-A1; and JP2007515689-W (2005).

${ }^{32}$ Produced by International Radiation Detectors, Inc., see www.ird-inc.com.

${ }^{33}$ P. Prigent, Ph. Hollander, M. Labat, M. E. Couprie, J. L. Marlats, C. Laulhé, J. Luning, T. Moreno, P. Morin, A. Nadji, F. Polack, S. Ravy, M. Silly, and F. Sirotti, "Progress on the femto-slicing project at the synchrotron SOLEIL," J. Phys.: Conf. Ser. 425, 072022 (2013). 